\title{
Exponential Inequalities for Positively Associated Random Variables and Applications
}

\author{
Guodong Xing, ${ }^{1}$ Shanchao Yang, ${ }^{2}$ and Ailin Liu ${ }^{3}$ \\ ${ }^{1}$ Department of Mathematics, Hunan University of Science and Engineering, Yongzhou, \\ 425100 Hunan, China \\ ${ }^{2}$ Department of Mathematics, Guangxi Normal University, Guilin, 541004 Guangxi, China \\ ${ }^{3}$ Department of Physics, Hunan University of Science and Engineering, Yongzhou, \\ 425100 Hunan, China
}

Correspondence should be addressed to Guodong Xing, xingguod@163.com

Received 1 January 2008; Accepted 6 March 2008

Recommended by Jewgeni Dshalalow

We establish some exponential inequalities for positively associated random variables without the boundedness assumption. These inequalities improve the corresponding results obtained by Oliveira (2005). By one of the inequalities, we obtain the convergence rate $n^{-1 / 2}(\log \log n)^{1 / 2}(\log n)^{2}$ for the case of geometrically decreasing covariances, which closes to the optimal achievable convergence rate for independent random variables under the Hartman-Wintner law of the iterated logarithm and improves the convergence rate $n^{-1 / 3}(\log n)^{5 / 3}$ derived by Oliveira (2005) for the above case.

Copyright ( 2008 Guodong Xing et al. This is an open access article distributed under the Creative Commons Attribution License, which permits unrestricted use, distribution, and reproduction in any medium, provided the original work is properly cited.

\section{Introduction}

A finite family of random variables $\left\{X_{i}, 1 \leq i \leq n\right\}$ is said to be positively associated (PA) if for every pair of disjoint subsets $A_{1}$ and $A_{2}$ of $\{1,2, \ldots, n\}$,

$$
\operatorname{Cov}\left\{f_{1}\left(X_{i}, i \in A_{1}\right), f_{2}\left(X_{j}, j \in A_{2}\right)\right\} \geq 0
$$

whenever $f_{1}$ and $f_{2}$ are coordinatewise increasing and the covariance exists. An infinite family is positively associated if every finite subfamily is positively associated.

The exponential inequalities and moment inequalities for partial sum $\sum_{i=1}^{n}\left(X_{i}-E X_{i}\right)$ play a very important role in various proofs of limit theorems. For positively associated random variables, Birkel [1] seems the first to get some moment inequalities. Shao and $\mathrm{Yu}$ [2] generalized later the previous results. Recently, Ioannides and Roussas [3] established a Bernstein-Hoeffding-type inequality for stationary and positively associated random variables being bounded; and Oliveira [4] gave a similar inequality dropping the boundedness 
assumption by the existence of Laplace transforms. By the inequality, he obtained that the rate of $\sum_{i=1}^{n}\left(X_{i}-E X_{i}\right) / n \rightarrow 0$ a.s. is $n^{-1 / 3}(\log n)^{5 / 3}$ under the rate of covariances supposed to be geometrically decreasing, that is, $\rho^{n}$ for some $0<\rho<1$. The convergence rate is partially improved by Yang and Chen [5] only for positively associated random variables being bounded. Furthermore, the rate of convergence in [4] is even lower than that obtained by [3]. These motivate us to establish some new exponential inequalities in order to improve the inequalities and the convergence rate which [4] obtained without the boundedness assumption. It is the main purpose of this paper. Our inequalities in Sections 3-5 improve the corresponding results in [4]. Moreover, by Corollary 5.4 (which can be seen in Section 5), we may get the rate $n^{-1 / 2}(\log \log n)^{1 / 2}(\log n)^{2}$ if the rate of covariances is geometrically decreasing. The result closes to the optimal achievable convergence rate for independent random variables under the Hartman-Wintner law of the iterated logarithm and improves the relevant result obtained by [4] without the boundedness assumption.

Throughout this paper, we always suppose that $C$ denotes a positive constant which only depends on some given numbers, $[x]$ denotes the integral of $x$; and this paper is organized as follows. Section 2 contains some lemmas used later in the proof of theorems, and some notations. Section 3 studies the truncated part giving conditions on the truncating sequence to enable the proof of some exponential inequalities for these terms. Section 4 treats the tails left aside from the truncation. Section 5 summarizes the partial results into some theorems and gives some applications.

\section{Some lemmas and notations}

Firstly, we quote two lemmas as follows.

Lemma 2.1 (see [6]). Let $\left\{X_{i}, 1 \leq i \leq n\right\}$ be positively associated random variables bounded by a constant $M$. Then for any $\lambda>0$,

$$
\left|E\left(\exp \left(\lambda \sum_{i=1}^{n} X_{i}\right)\right)-\prod_{i=1}^{n} E\left(\exp \left(\lambda X_{i}\right)\right)\right| \leq \lambda^{2} \exp (n \lambda M) \sum_{1 \leq i<j \leq n} \operatorname{Cov}\left(X_{i}, X_{j}\right) .
$$

Lemma 2.2 (see [7]). Let $\left\{X_{i}, i \geq 1\right\}$ be a positively associated sequence with zero mean and

$$
\sum_{i=1}^{\infty} v^{1 / 2}\left(2^{i}\right)<\infty
$$

where $v(n)=\sup _{i \geq 1} \sum_{j: j-i \geq n} \operatorname{Cov}^{1 / 2}\left(X_{i}, X_{j}\right)$. Then there exists a positive constant $C$ such that

$$
E \max _{1 \leq j \leq n}\left|\sum_{i=1}^{j} X_{i}\right|^{2} \leq \operatorname{Cn}\left\{\sup _{i \geq 1} E X_{i}^{2}+\left(\sup _{i \geq 1} E X_{i}^{2}\right)^{1 / 2}\right\}
$$

Remark 2.3 (see condition (2.2) is quite weak). In fact, it is satisfied only if $v(n) \leq$ $C(\log n)^{-2}(\log \log n)^{-2-\xi}$ for some $\xi>0$. So it is weaker than the corresponding condition in $[1,2]$. 
For the formulation of the assumptions to be made in this paper, some notations are required. Thus let $c_{n}, n \geq 1$ be a sequence of nonnegative real numbers such that $c_{n} \rightarrow \infty$ and $u(n)=\sup _{i \geq 1} \sum_{j: j-i \geq n} \operatorname{Cov}\left(X_{i}, X_{j}\right)$. Also, for convenience, we define $X_{n i}$ by $X_{n i}=X_{i}$ for $1 \leq i \leq n$ and $X_{n i}=0$ for $i>n$, and let

$$
\begin{aligned}
& X_{1, i, n}=-\frac{c_{n}}{2} I_{\left(-\infty,-c_{n} / 2\right)}\left(X_{n i}\right)+X_{n i} I_{\left(-c_{n} / 2, c_{n} / 2\right)}\left(X_{n i}\right)+\frac{c_{n}}{2} I_{\left(c_{n} / 2,+\infty\right)}\left(X_{n i}\right), \\
& X_{2, i, n}=\left(X_{n i}-\frac{c_{n}}{2}\right) I_{\left(c_{n} / 2,+\infty\right)}\left(X_{n i}\right), \quad X_{3, i, n}=\left(X_{n i}+\frac{c_{n}}{2}\right) I_{\left(-\infty,-c_{n} / 2\right)}\left(X_{n i}\right),
\end{aligned}
$$

for each $n, i \geq 1$, where $I_{A}$ represents the characteristic function of the set $A$. Consider now a sequence of natural numbers $p_{n}$ such that for each $n \geq 1, p_{n}<n / 2$, and set $r_{n}=\left[n /\left(2 p_{n}\right)\right]+1$. Define, then,

$$
Y_{q, j, n}=\sum_{i=2(j-1) p_{n}+1}^{2(j-1) p_{n}+p_{n}}\left(X_{q, i, n}-E\left(X_{q, i, n}\right)\right), \quad Z_{q, j, n}=\sum_{i=2(j-1) p_{n}+p_{n}+1}^{2 j p_{n}}\left(X_{q, i, n}-E\left(X_{q, i, n}\right)\right)
$$

for $q=1,2,3, j=1,2, \ldots, r_{n}$, and

$$
S_{q, n, o d}=\sum_{j=1}^{r_{n}} Y_{q, j, n}, \quad S_{q, n, e v}=\sum_{j=1}^{r_{n}} Z_{q, j, n}
$$

Clearly, $n \leq 2 r_{n} p_{n}<2 n$.

The proofs given later will be divided into the control of the bounded terms that correspond to the index $q=1$ and the control of the unbounded terms, corresponding to the indices $q=2,3$.

\section{Control of the bounded terms}

In this section, we will work hard to control the bounded terms. For this purpose, we give some lemmas as follows.

Lemma 3.1. Let $\left\{X_{i}, i \geq 1\right\}$ be a positively associated sequence. Then on account of definitions (2.5), (2.6), (2.7), and for every $\lambda>0$,

$$
\begin{aligned}
& \left|E\left(\exp \left(\lambda S_{1, n, o d}\right)\right)-\prod_{j=1}^{r_{n}} E\left(\exp \left(\lambda Y_{1, j, n}\right)\right)\right| \leq \lambda^{2} n u\left(p_{n}\right) \exp \left(\lambda n c_{n}\right), \\
& \left|E\left(\exp \left(\lambda S_{1, n, e v}\right)\right)-\prod_{j=1}^{r_{n}} E\left(\exp \left(\lambda Z_{1, j, n}\right)\right)\right| \leq \lambda^{2} n u\left(p_{n}\right) \exp \left(\lambda n c_{n}\right) .
\end{aligned}
$$

Proof. Similarly to the proof of Lemma 3.2 in [4], it is omitted here. 
Lemma 3.2. Let $\left\{X_{i}, i \geq 1\right\}$ be a positively associated sequence and let (2.2) hold. If $0<\lambda p_{n} c_{n} \leq 1$ for $\lambda>0$, then

$$
\begin{aligned}
& \prod_{j=1}^{r_{n}} E\left(\exp \left(\lambda Y_{1, j, n}\right)\right) \leq \exp \left(C_{1} \lambda^{2} n c_{n}^{2}\right), \\
& \prod_{j=1}^{r_{n}} E\left(\exp \left(\lambda Z_{1, j, n}\right)\right) \leq \exp \left(C_{1} \lambda^{2} n c_{n}^{2}\right),
\end{aligned}
$$

where $C_{1}$ is a constant, not depending on $n$.

Proof. Since $E Y_{1, j, n}=0$ and $0<\lambda p_{n} c_{n} \leq 1$, we may have

$$
\begin{aligned}
E\left(\exp \left(\lambda Y_{1, j, n}\right)\right) & =\sum_{k=0}^{\infty} \frac{E\left(\lambda Y_{1, j, n}\right)^{k}}{k !}=1+\sum_{k=2}^{\infty} \frac{E\left(\lambda Y_{1, j, n}\right)^{k}}{k !} \\
& \leq 1+E\left(\lambda Y_{1, j, n}\right)^{2} \sum_{k=2}^{\infty} \frac{1}{k !} \leq 1+\lambda^{2} E Y_{1, j, n}^{2} \leq \exp \left(\lambda^{2} E Y_{1, j, n}^{2}\right)
\end{aligned}
$$

By this, Lemma 2.2 and $\left|X_{1, i, n}\right| \leq c_{n} / 2$,

$$
\begin{aligned}
\prod_{j=1}^{r_{n}} E\left(\exp \left(\lambda Y_{1, j, n}\right)\right) & \leq \exp \left(\lambda^{2} \sum_{j=1}^{r_{n}} E Y_{1, j, n}^{2}\right) \\
& \leq \exp \left(C \lambda^{2} p_{n} \sum_{j=1}^{r_{n}}\left\{\sup _{i \geq 1} \operatorname{Var}\left(X_{1, i, n}\right)+\left(\sup _{i \geq 1} \operatorname{Var}\left(X_{1, i, n}\right)\right)^{1 / 2}\right\}\right) \\
& \leq \exp \left(C \lambda^{2} p_{n} \sum_{j=1}^{r_{n}}\left\{\sup _{i \geq 1} E X_{1, i, n}^{2}+\left(\sup _{i \geq 1} E X_{1, i, n}^{2}\right)^{1 / 2}\right\}\right) \\
& \leq \exp \left(C \lambda^{2} r_{n} p_{n}\left(c_{n} / 2\right)^{2}\right) \leq \exp \left(C_{1} \lambda^{2} n c_{n}^{2}\right)
\end{aligned}
$$

as desired. The proof is completed.

Remark 3.3. The upper bound of [4, Lemma 3.1] is $\exp \left(\lambda^{2} n p_{n} c_{n}^{2}\right)$, and so the upper bound of Lemma 3.1 is much sharper than that of [4] when $p_{n} \rightarrow \infty$, this is the reason why we choose the condition $0<\lambda p_{n} c_{n} \leq 1$, which is equivalent to $0<\lambda \leq 1 /\left(p_{n} c_{n}\right)$ and enables us to get the desired upper bound by Lemma 2.2.

Combining Lemmas 3.1 and 3.2 yields easily the following result.

Lemma 3.4. Let $\left\{X_{i}, i \geq 1\right\}$ be a positively associated sequence and let (2.2) hold. If $0<\lambda p_{n} c_{n} \leq 1$ for $\lambda>0$, then for any $\varepsilon>0$,

$$
P\left(\left|\sum_{i=1}^{n}\left(X_{1, i, n}-E X_{1, i, n}\right)\right|>n \varepsilon\right) \leq 4\left\{\lambda^{2} n u\left(p_{n}\right) e^{\lambda n c_{n}}+e^{C_{1} \lambda^{2} n c_{n}^{2}}\right\} e^{-n \lambda \varepsilon / 2},
$$

where $X_{1, i, n}$ and $C_{1}$ are just as in (2.5) and (3.2).

By Lemma 3.4, one can show a result as follows. 
Theorem 3.5. Let $\left\{X_{i}, i \geq 1\right\}$ be a positively associated sequence and let (2.2) hold. Suppose that $p_{n} \leq n / \alpha \log n$ for some $\alpha>0, p_{n} \rightarrow \infty$, and

$$
\frac{\log n}{n^{2 \alpha / 3} p_{n} c_{n}^{2}} \exp \left\{\left(\frac{\alpha n \log n}{p_{n}}\right)^{1 / 2}\right\} u\left(p_{n}\right) \leq C_{0}<\infty,
$$

where $C_{0}$ is a constant which does not depend on $n$. Set $\varepsilon_{n}=(10 / 3)\left(\alpha p_{n} c_{n}^{2} \log n / n\right)^{1 / 2}$. Then there exists a positive constant $C_{2}$, which only depends on $\alpha>0$, such that

$$
P\left(\left|\sum_{i=1}^{n}\left(X_{1, i, n}-E X_{1, i, n}\right)\right|>n \varepsilon_{n}\right) \leq C_{2} \exp (-\alpha \log n) .
$$

Proof. Let $\lambda=10 \alpha \log n / 3 n \varepsilon_{n}=\left(\alpha \log n / n p_{n} c_{n}^{2}\right)^{1 / 2}$ and $\varepsilon=\varepsilon_{n}$ in Lemma 3.4. Then it is obvious that $\lambda p_{n} c_{n} \leq 1$ from $p_{n} \leq n / \alpha \log n$ and that

$$
e^{-n \lambda \varepsilon_{n} / 2}=e^{-(5 / 3) \alpha \log n}
$$

Noting that $p_{n} \rightarrow \infty$, we may have

$$
\begin{gathered}
e^{C_{1} \lambda^{2} n c_{n}^{2}}=\exp \left(\frac{C_{1} \alpha \log n}{p_{n}}\right) \leq \exp \left(\frac{2}{3} \alpha \log n\right), \\
\lambda^{2} n u\left(p_{n}\right) e^{\lambda n c_{n}}=\frac{\alpha \log n}{p_{n} c_{n}^{2}} \exp \left\{\left(\frac{\alpha n \log n}{p_{n}}\right)^{1 / 2}\right\} u\left(p_{n}\right) \\
\leq C_{2} n^{2 \alpha / 3}=C_{2} \exp \left(\frac{2}{3} \alpha \log n\right)
\end{gathered}
$$

by (3.7). Combining (3.9)-(3.11), we can get (3.8) by Lemma 3.4. The proof is completed.

Remark 3.6. (1) Let us compare Theorem 3.5 with [4, Theorem 3.6]. Our result drops the strict stationarity of the positively associated random variables; and to obtain (3.8), Oliveira [4] used the following condition:

$$
\frac{\log n}{p_{n} c_{n}^{2}} \exp \left\{\left(\frac{\alpha n \log n}{p_{n}}\right)^{1 / 2}\right\} u\left(p_{n}\right) \leq C_{0}<\infty .
$$

Obviously, (3.7) is weaker than (3.12).

(2) Although Theorem 3.5 holds under weaker conditions, it cannot make us get a much faster convergence rate for the almost sure convergence to zero of $\sum_{i=1}^{n}\left(X_{i}-E X_{i}\right) / n$ than the one of convergence in [4]. This is because $\varepsilon_{n}=(10 / 3)\left(\alpha p_{n} c_{n}^{2} \log n / n\right)^{1 / 2}$, preventing us from getting the convergence rate $n^{-1 / 2}(\log \log n)^{1 / 2}(\log n)^{2}$ for the case of geometrically decreasing covariances. So to obtain the above rate, we show another exponential inequality (3.20) in which $\varepsilon_{n}=p_{n} c_{n} \sqrt{\log \log n} \log n / 2 n$, permitting us to get the desired rate when we use condition (3.19) instead of condition (3.7), which is weaker than condition (3.19) for the case $\alpha>2 / 3$, $0<\delta<1 / 2$, and $p_{n} \leq(4+3 \delta)^{2} n / \alpha \epsilon^{2} \log n \log \log n$. 
Now, let us consider (3.8) again. By Borel-Cantelli lemma, we need $\sum_{n=1}^{\infty} e^{-\alpha \log n}<\infty$ for some $\alpha>0$ in order to get strong law of large numbers. However, it is not true for $0<\alpha \leq 1$. To avoid this case, we show another exponential inequality.

Theorem 3.7. Let $\left\{X_{i}, i \geq 1\right\}$ be a positively associated sequence and let (2.2) hold. Assume that $\left\{\varepsilon_{n}: n \geq 1\right\}$ is a positive real sequence which satisfies

$$
\frac{p_{n} c_{n} \log n}{n \varepsilon_{n}} \longrightarrow 0, \quad \frac{c_{n}^{2} \log n}{n \varepsilon_{n}^{2}} \longrightarrow 0,
$$

and for some $\epsilon>0$ and $\delta>0$,

$$
n^{-(1+2 \delta)}\left(\frac{\log n}{\varepsilon_{n}}\right)^{2} \exp \left(\frac{2(1+3 \delta) c_{n} \log n}{\varepsilon_{n} \epsilon}\right) u\left(p_{n}\right) \leq C_{0}<\infty .
$$

Then there exists a positive constant $C$, which depends on $\epsilon>0$ and $\delta>0$, such that

$$
P\left(\left|\sum_{i=1}^{n}\left(X_{1, i, n}-E X_{1, i, n}\right)\right|>n \varepsilon_{n} \epsilon\right) \leq C \exp (-(1+\delta) \log n) .
$$

Proof. Let $\lambda=2(1+3 \delta) \log n / n \varepsilon_{n} \epsilon$ and let $\varepsilon=\varepsilon_{n} \epsilon$ in Lemma 3.4. Then it is obvious that $\lambda p_{n} c_{n} \leq$ 1 from (3.13) and that

$$
e^{-n \curlywedge \varepsilon / 2}=e^{-n \lambda \varepsilon_{n} \epsilon / 2}=e^{-(1+3 \delta) \log n}
$$

Also, we can get that

$$
e^{C_{1} \lambda^{2} n c_{n}^{2}}=\exp \left(\frac{C_{1} 4(1+3 \delta)^{2} c_{n}^{2} \log n}{\epsilon^{2} n \varepsilon_{n}^{2}}\right) \leq \exp (2 \delta \log n)
$$

by (3.13), and that

$$
\begin{aligned}
\lambda^{2} n u\left(p_{n}\right) e^{\lambda n c_{n}} & =\left(\frac{2(1+3 \delta)}{\epsilon}\right)^{2}\left(\frac{\log n}{\varepsilon_{n}}\right)^{2} n^{-1} \exp \left(\frac{2(1+3 \delta) c_{n} \log n}{\varepsilon_{n} \epsilon}\right) u\left(p_{n}\right) \\
& \leq C n^{2 \delta}=C \exp (2 \delta \log n)
\end{aligned}
$$

by (3.14). Combining (3.16)-(3.18), we can obtain (3.15) by Lemma 3.4. result.

Taking $\varepsilon_{n}=p_{n} c_{n} \sqrt{\log \log n} \log n / 2 n$ in Theorem 3.7, we can get easily the following

Corollary 3.8. Let $\left\{X_{i}, i \geq 1\right\}$ be a positively associated sequence and let (2.2) hold. Suppose that $p_{n}$ satisfies $\sqrt{n / \log n} \leq p_{n}<n / 2$ and for some $\epsilon>0$ and $\delta>0$,

$$
\frac{n^{1-2 \delta}}{p_{n}^{2} c_{n}^{2} \log \log n} \exp \left(\frac{4(1+3 \delta) n}{\epsilon p_{n} \sqrt{\log \log n}}\right) u\left(p_{n}\right) \leq C_{0}<\infty .
$$


Then there exists a positive constant $C_{3}$, which depends on $\epsilon>0$ and $\delta>0$, such that

$$
P\left(\left|\sum_{i=1}^{n}\left(X_{1, i, n}-E X_{1, i, n}\right)\right|>\epsilon p_{n} c_{n} \sqrt{\log \log n} \log n\right) \leq C_{3} \exp (-(1+\delta) \log n) .
$$

\section{Control of the unbounded terms}

In this section, we will try ourselves to control the unbounded terms. Firstly, it is obvious that the variables $X_{2, i, n}$ and $X_{3, i, n}$ are positively associated but not bounded, even for fixed $n$. This means that Lemma 3.1 cannot be applied to the sum of such terms. While we may note that these variables depend only on the tails of distribution of the original variables. Hence by controlling the decrease rate of these tails, we may give some exponential inequalities for the sums of $X_{2, i, n}$ or $X_{3, i, n}$. The results we get are listed below.

Lemma 4.1. Let $\left\{X_{i}, i \geq 1\right\}$ be a positively associated sequence that satisfies

$$
\sup _{i \geq 1,|t| \leq \omega} E\left(e^{t X_{i}}\right) \leq M_{\omega}<\infty
$$

for some $\omega>0$ and let (2.2) hold. Then for $0<t \leq \omega$,

$$
P\left(\max _{1 \leq j \leq n}\left|\sum_{i=1}^{j}\left(X_{q, i, n}-E X_{q, i, n}\right)\right|>n \varepsilon\right) \leq \frac{C \sqrt{2 M_{\omega} e^{-t c_{n} / 2}}}{n t \varepsilon^{2}}, \quad q=2,3 .
$$

Proof. Firstly, let us estimate $E X_{q, i, n}^{2}$. Without loss of generality, set $q=2$. We will assume $F(x)=$ $P\left(X_{i}>x\right)$. Then by Markov's inequality and $\sup _{i \geq 1,|t| \leq \omega} E\left(e^{t X_{i}}\right) \leq M_{\omega}<\infty$ for some $\omega>0$, it follows that, for $0<t \leq \omega$,

$$
F(x) \leq e^{-t x} E\left(e^{t X_{i}}\right) \leq M_{\omega} e^{-t x} .
$$

Writing the mathematical expectation as a Stieltjes integral and integrating by parts, we have

$$
\begin{aligned}
E X_{2, i, n}^{2} & =-\int_{\left(c_{n} / 2,+\infty\right)}\left(x-\frac{c_{n}}{2}\right)^{2} d F(x) \\
& =-\left.\left(x-\frac{c_{n}}{2}\right)^{2} F(x)\right|_{c_{n} / 2} ^{+\infty}+\int_{\left(c_{n} / 2,+\infty\right)} 2\left(x-\frac{c_{n}}{2}\right) F(x) d x \\
& =-\lim _{x \rightarrow+\infty}\left(x-\frac{c_{n}}{2}\right)^{2} F(x)+\int_{\left(c_{n} / 2,+\infty\right)} 2\left(x-\frac{c_{n}}{2}\right) F(x) d x \\
& =\int_{\left(c_{n} / 2,+\infty\right)} 2\left(x-\frac{c_{n}}{2}\right) F(x) d x \\
& \leq 2 M_{\omega} \int_{\left(c_{n} / 2,+\infty\right)}\left(x-\frac{c_{n}}{2}\right) e^{-t x} d x \\
& =2 M_{\omega} \frac{e^{-t c_{n} / 2}}{t^{2}}
\end{aligned}
$$


by the inequality stated earlier. Hence using (4.4) and Lemma 2.2, we have, for $n$ large enough,

$$
\begin{aligned}
P\left(\max _{1 \leq j \leq n}\left|\sum_{i=1}^{j}\left(X_{2, i, n}-E X_{2, i, n}\right)\right|>n \varepsilon\right) & \leq \frac{E \max _{1 \leq j \leq n}\left|\sum_{i=1}^{j}\left(X_{2, i, n}-E X_{2, i, n}\right)\right|^{2}}{n^{2} \varepsilon^{2}} \\
& \leq \frac{C n\left\{\sup _{i \geq 1} \operatorname{Var}\left(X_{2, i, n}\right)+\left(\sup _{i \geq 1} \operatorname{Var}\left(X_{2, i, n}\right)\right)^{1 / 2}\right\}}{n^{2} \varepsilon^{2}} \\
& \leq \frac{C\left\{\sup _{i \geq 1} E X_{2, i, n}^{2}+\left(\sup _{i \geq 1} E X_{2, i, n}^{2}\right)^{1 / 2}\right\}}{n \varepsilon^{2}} \\
& \leq \frac{C \sqrt{2 M_{\omega} e^{-t c_{n} / 2}}}{n t \varepsilon^{2}}
\end{aligned}
$$

This completes the proof of the lemma.

Remark 4.2. Let $\left\{X_{i}, i \geq 1\right\}$ be a positively associated sequence and let (2.2) hold (as mentioned above, it is a quite weak condition). Then Lemma 4.1 improves the corresponding result in [4] from the following aspects.

(i) The assumption of the stationarity of $\left\{X_{i}, i \geq 1\right\}$ is dropped.

(ii) The sum in (4.2) is

$$
\max _{1 \leq j \leq n}\left|\sum_{i=1}^{j}\left(X_{q, i, n}-E X_{q, i, n}\right)\right|, \operatorname{not}\left|\sum_{i=1}^{n}\left(X_{q, i, n}-E X_{q, i, n}\right)\right| \text { in [4]. }
$$

(iii) The upper bound of the exponential inequality in [4, Lemma 4.1] is $2 M_{\omega} n e^{-t \widetilde{c_{n}}} / t^{2} \varepsilon^{2}$, where $\tilde{c_{n}} \rightarrow \infty$. So, assuming $c_{n}=4 \widetilde{c_{n}}$ in the inequality (4.2), we can obtain that the upper bound of our inequality is $C \sqrt{2 M_{\omega}} e^{-t \widetilde{c_{n}}} / n t^{2} \varepsilon^{2}$. Obviously, $C \sqrt{2 M_{\omega}} e^{-t \widetilde{c_{n}}} / n t^{2} \varepsilon^{2} \leq$ $2 M_{\omega} n e^{-t \widetilde{c_{n}}} / t^{2} \varepsilon^{2}$ for sufficiently large $n$. That is, the upper bound in Lemma 4.1 is much lower than that of [4, Lemma 4.1]. $t$ and $c_{n}$.

Applying Lemma 4.1, one can get immediately the following result by taking values for

Corollary 4.3. Let $\left\{X_{i}, i \geq 1\right\}$ be a positively associated sequence that satisfies $\sup _{i \geq 1,|t| \leq \omega} E\left(e^{t X_{i}}\right) \leq$ $M_{\omega}<\infty$ for some $\omega>0$ and let (2.2) hold. Then

$$
P\left(\max _{1 \leq j \leq n}\left|\sum_{i=1}^{j}\left(X_{q, i, n}-E X_{q, i, n}\right)\right|>n \varepsilon\right) \leq \frac{C \sqrt{2 M_{\omega}}}{2 \alpha n \varepsilon^{2}} \exp (-\alpha \log n), \quad q=2,3,
$$

provided $t=2 \alpha$ and $c_{n}=2 \log n$, and

$$
P\left(\max _{1 \leq j \leq n}\left|\sum_{i=1}^{j}\left(X_{q, i, n}-E X_{q, i, n}\right)\right|>n \varepsilon\right) \leq \frac{C \sqrt{2 M_{\omega}}}{2 \alpha n \varepsilon^{2}} \exp (-(1+\delta) \log n), \quad q=2,3,
$$

provided $t=2 \alpha$ and $c_{n}=(2(1+\delta) / \alpha) \log n$, where $\alpha$ and $\delta$ are as in (3.8) and (3.13). 


\section{Strong convergences and rates}

This section summarizes the results stated earlier. In addition, we give a convergence rate for geometrically decreasing covariances, which improves the relevant one obtained by [4].

Theorem 5.1. Let $\left\{X_{i}, i \geq 1\right\}$ be a positively associated sequence satisfying

$$
\frac{1}{n^{2 \alpha / 3} p_{n} \log n} \exp \left\{\left(\frac{\alpha n \log n}{p_{n}}\right)^{1 / 2}\right\} u\left(p_{n}\right) \leq C_{0}<\infty
$$

for some $\alpha>0, n / \alpha \log n \geq p_{n} \rightarrow \infty$ and let (2.2) hold. Suppose that $\varepsilon_{n}$ is as in Theorem 3.5 and there exists $\omega>\alpha$ that satisfies $\sup _{i \geq 1,|t| \leq \omega} E\left(e^{t X_{i}}\right) \leq M_{\omega}<\infty$. Then for sufficiently large $n$,

$$
P\left(\left|\sum_{i=1}^{n}\left(X_{i}-E X_{i}\right)\right|>3 n \varepsilon_{n}\right) \leq\left(C_{2}+\frac{9 C \sqrt{2 M_{\omega}}}{200 \alpha^{2} p_{n} \log ^{3} n}\right) \exp (-\alpha \log n) .
$$

Proof. Combining Theorem 3.5 and Corollary 4.3 yields the desired result (5.2).

Remark 5.2. Theorem 5.1 improves [4, Theorem 5.1], because the latter uses the following more restrictive conditions.

(i) $\left\{X_{i}, i \geq 1\right\}$ is a strictly stationary sequence.

(ii) $\left\{X_{i}, i \geq 1\right\}$ satisfies $\left(1 / p_{n} \log n\right) \exp \left\{\left(\alpha n \log n / p_{n}\right)^{1 / 2}\right\} u\left(p_{n}\right) \leq C_{0}<\infty$. Clearly, it implies (5.1).

(iii) The latter has a higher upper bound than our result, because $9 C \sqrt{2 M_{\omega}} /$ $200 \alpha^{2} p_{n} \log ^{3} n \leq 2 M_{\omega} n^{2} / 9 \alpha^{3} p_{n} \log ^{3} n$ for sufficiently large $n$.

Combining Corollaries 3.8 and 4.3, we may get easily the following result.

Theorem 5.3. Let $\left\{X_{i}, i \geq 1\right\}$ be a positively associated sequence satisfying (3.19) for $\sqrt{n / \log n} \leq$ $p_{n}<n / 2$, some $\epsilon>0$, and $\delta>0$ and let (2.2) hold. Suppose that $\sup _{i \geq 1,|t| \leq \omega} E\left(e^{t X_{i}}\right) \leq M_{\omega}<\infty$ for some $\omega>\alpha$. Then for $n$ large enough,

$$
P\left(\left|\sum_{i=1}^{n}\left(X_{i}-E X_{i}\right)\right|>3 n \epsilon_{n}\right) \leq\left(C_{3}+\frac{C \sqrt{2 M_{\omega}}}{2 \alpha n \epsilon_{n}^{2}}\right) \exp (-(1+\delta) \log n)
$$

where $\epsilon_{n}=\epsilon p_{n} c_{n} \sqrt{\log \log n} \log n / n$ and $c_{n}=(2(1+\delta) / \alpha) \log n$.

Applying Theorem 5.3, one may have immediately some strong laws of large numbers by taking $p_{n}=[\sqrt{n}]$ and $p_{n}=[n / 4]$, respectively.

Corollary 5.4. Let $\left\{X_{i}, i \geq 1\right\}$ be a positively associated sequence which satisfies $\sup _{i \geq 1,|t| \leq \omega} E\left(e^{t X_{i}}\right) \leq M_{\omega}<\infty$ for some $\omega>\alpha$. Then

$$
\frac{\sum_{i=1}^{n}\left(X_{i}-E X_{i}\right)}{\sqrt{n \log \log n \log ^{2} n}} \longrightarrow 0, \text { a.s., }
$$


provided that

$$
\frac{\exp (\alpha \sqrt{n}) u([\sqrt{n}])}{n^{2 \delta} \log ^{2} n \log \log n} \leq C<\infty \quad \text { for some } \alpha>0, \delta>0
$$

and (2.2) holds; and

$$
\frac{\sum_{i=1}^{n}\left(X_{i}-E X_{i}\right)}{n \sqrt{\log \log n} \log ^{2} n} \longrightarrow 0, \text { a.s., }
$$

provided that

$$
\frac{u([n / 4])}{n^{1+2 \delta} \log ^{2} n \log \log n} \leq C<\infty \quad \text { for some } \delta>0
$$

and (2.2) holds.

Finally, one gives some applications of Corollary 5.4.

(1) Suppose now $\operatorname{Cov}\left(X_{i}, X_{j}\right)=C \rho^{|i-j|}$ for some $0<\rho<1$. Then $v([\sqrt{n}]) \sim C \rho^{\sqrt{n} / 2}$ and $u([\sqrt{n}]) \sim C \rho^{\sqrt{n}}$, so (2.2) is satisfied and

$$
\exp (\alpha \sqrt{n}) u([\sqrt{n}]) \sim C\left(\rho e^{\alpha}\right)^{\sqrt{n}} \longrightarrow 0
$$

by choosing $\alpha>0$ with $0<\rho e^{\alpha}<1$. This means that one requires only $0<\alpha<-\log \rho$, not $\alpha>$ $8 / 3$ in [4]. It is due to Lemma 4.1. By (5.8), one knows that (5.5) holds. Hence one gets finally that $\sum_{i=1}^{n}\left(X_{i}-E X_{i}\right) / n \rightarrow 0$, a.s., converges at the rate $n^{-1 / 2}(\log \log n)^{1 / 2} \log ^{2} n$ which closes to the optimal achievable convergence rate for independent random variables under the Hartman-Wintner law of the iterated logarithm. However, Oliveira [4] only got $n^{-1 / 3} \log ^{5 / 3} n$ for the case mentioned above. Clearly, the convergence rate is much lower than ours.

(2) If $\operatorname{Cov}\left(X_{i}, X_{j}\right)=C|j-i|^{-\tau}$ for some $\tau>2$, or $\operatorname{Cov}\left(X_{i}, X_{j}\right)=C|j-i|^{-2} \log ^{-\eta}|j-i|$ for some $\eta>8$, then it is clear that (5.7) and (2.2) can be satisfied. Therefore By (5.6), one does have almost sure convergence but without rates. The explicit reason could be seen in [4].

\section{Acknowledgments}

The authors thank the referees for their careful reading and valuable comments that improved presentation of the manuscript. This work is supported by the National Science Foundation of China (Grant no. 10161004), the Natural Science Foundation of Guangxi (Grant no. 0728091), and the key Science Foundation of Hunan University of Science and Engineering.

\section{References}

[1] T. Birkel, "Moment bounds for associated sequences," Annals of Probability, vol. 16, no. 3, pp. 1184-1193, 1988.

[2] Q.-M. Shao and H. Yu, "Weak convergence for weighted empirical processes of dependent sequences," Annals of Probability, vol. 24, no. 4, pp. 2098-2127, 1996.

[3] D. A. Ioannides and G. G. Roussas, "Exponential inequality for associated random variables," Statistics E Probability Letters, vol. 42, no. 4, pp. 423-431, 1999. 
[4] P. E. Oliveira, "An exponential inequality for associated variables," Statistics \& Probability Letters, vol. 73, no. 2, pp. 189-197, 2005.

[5] S.-C. Yang and M. Chen, "Exponential inequalities for associated random variables and strong laws of large numbers," Science in China A, vol. 50, no. 5, pp. 705-714, 2006.

[6] I. Dewan and B. L. S. Prakasa Rao, "A general method of density estimation for associated random variables," Journal of Nonparametric Statistics, vol. 10, no. 4, pp. 405-420, 1999.

[7] Y. S. Chao, "Complete convergence for sums of positively associated sequences," Chinese Journal of Applied Probability and Statistics, vol. 17, no. 2, pp. 197-202, 2001, Chinese. 